\title{
Compensation Voltage (CV) Peak Shapes Using a Domed FAIMS with the Inner Electrode Translated to Various Longitudinal Positions
}

\author{
Roger Guevremont and Govindanunny Thekkadath \\ Ionalytics Corporation, Ottawa, Ontario, Canada \\ Christopher K. Hilton \\ University of Florida, Gainesville, Florida, USA
}

High-field asymmetric waveform ion mobility spectrometry (FAIMS) separates ions at atmospheric pressure based on the difference in the mobility of an ion in a strong electric field and in a weak electric field. This field-dependent mobility of an ion is reflected in the compensation voltage $(\mathrm{CV})$ at which the ion is transmitted through FAIMS at an applied asymmetric waveform dispersion voltage (DV). In this report, we show that experimental CV peak shapes using dome tipped inner electrode FAIMS prototypes with inner/outer electrode radii of: (1) $0.2 / 0.4 \mathrm{~cm}$ and (2) $0.4 / 0.6 \mathrm{~cm}$ are a function of the longitudinal position of the inner electrode. Varying the longitudinal position of the inner electrode modifies the electric fields between the surfaces of the hemispherical shaped inner electrode and the outer electrode in the vicinity of the ion outlet. In this region the position-dependent electric field strength $(E / N)$ effectively forms a second tandem FAIMS analyzer region having differing ion separation properties. The final tandem FAIMS separation is the intersection of the CV windows of these two differing FAIMS separations and, therefore, the peak width in the CV scan is dependent on the longitudinal tip displacement (LTD) of the inner electrode. CV scans are shown for a LTD range of 0.14 to $0.4 \mathrm{~cm}$. These scans illustrate that it is possible to control the FAIMS resolution ( $\mathrm{CV} /$ peak width) from about 1 for the $0.2 / 0.4 \mathrm{~cm}$ electrode set at intermediate longitudinal position to over 10 at the narrowest distance between the inner electrode and the ion outlet. (J Am Soc Mass Spectrom 2005, 16, 948-956) @ 2005 American Society for Mass Spectrometry

$\mathrm{H}$ igh-field asymmetric waveform ion mobility spectrometry (FAIMS) is a technology that separates ions at atmospheric pressure based on the difference in the mobility of an ion in strong and weak electric fields [1-14]. Fortuitously, the separation in FAIMS is based on different physical effects than the separation of conventional drift tube ion mobility spectrometry, as well as being independent of the $\mathrm{m} / \mathrm{z}$ of the ion measured by mass spectrometry [13]. In general, a tandem separation system such as LC-FAIMS-MS offers a powerful separation capability because the mechanism of separation of each device is independent. Such a tandem system composed of liquid chromatography, FAIMS, and mass spectrometry (LC-FAIMS-MS) has been applied to the separation of peptides from a tryptic digestion [15].

FAIMS has been shown to be a powerful ion processing tool for improving the detection limits of quan-

Published online April 26, 2005

Address reprint requests to Dr. R. Guevremont, Ionalytics Corporation, Building M50-IPF, 1200 Montreal Road, Ottawa, Ontario K1A 0R6, Canada.

E-mail: roger.guevremont@ionalyutics.com titative analysis using mass spectrometry, particularly for ion sources characterized by a high intensity of background ions. Analytical applications ranging from detection of inorganic species to the study of protein conformers have been demonstrated [9, 10, 13, 16-20]. A recent review [21] serves as an introduction to FAIMS literature, both in the areas of physical chemistry and applications.

In a recent paper, Smith and coworkers have undertaken a study of the resolution and sensitivity of FAIMS [22], making comparisons between experimental data and the results of ion trajectory computations. Shvartsburg et al. [23] have also studied the behavior of several ions in gas mixtures, and compared experimental results with predictions based on the theory of ion mobility in mixtures of gases [24]. Shvartsburg et al. suggest that significant improvements of separations utilizing FAIMS are possible using pairs of gases with widely disparate properties, one example cited being mixtures of $\mathrm{He}_{\mathrm{SF}}$. Further work remains to establish the practical applicability of this unusual mixture of gases. Models based on the field-dependence of the 
formation and dissociation of labile complexes $[25,26]$ have been proposed to qualitatively explain the behavior of an ion in these unusual gas mixtures, but these proposals remain to be theoretically or experimentally substantiated. More recently Smith and coworkers studied the impact of the design and the operating parameters on the optimization of FAIMS analyzers [23]. The theory of the impact of several experimental parameters, including the width of the analytical gap, waveform shape and frequency, and gas flow speed on FAIMS performance were investigated.

The practical application of FAIMS as an ion processing tool for mass spectrometry may require the design of electrodes with ion transmission and ion separation characteristics tuned for solving particular analytical problems. For example, the separation of conformers of proteins and the separation of isomers may require optimized resolution, whereas the analysis of tryptic fragments of a protein digest may be better served by a device capable of high sensitivity and high signal-tobackground ratio, but having sufficiently low resolution that a maximum number of multiply charged ions can be simultaneously detected without stepping the CV [15]. A recent study has suggested that such targeted performance is feasible, showing that the diameter of the electrodes in FAIMS is a key mechanical parameter controlling the resolution and sensitivity of FAIMS [27].

In this report, we consider the impact of the longitudinal location of the dome tipped inner electrode on the performance of FAIMS. The effect of the LTD on resolution and sensitivity was investigated for two prototypes of domed electrode FAIMS having inner/ outer electrode radii of $0.2 / 0.4 \mathrm{~cm}$ and $0.4 / 0.6 \mathrm{~cm}$.

\section{Experimental}

Figure 1 illustrates a cross section view of a domed electrode version of FAIMS in which the inner cylindrical electrode is terminated as a hemisphere and the outer electrode has a cylindrical inner surface, also terminated in a hemispherical shape. For this study, experimental measurements were taken with two FAIMS prototypes with inner/outer cylinder radii of $0.2 / 0.4$ and $0.4 / 0.6 \mathrm{~cm}$. In the domed device shown in Figure 1, the ions were first separated in a $0.2 \mathrm{~cm}$ wide annular space between the concentric cylinders along a region of the electrodes in which the inner and outer electrode surfaces are parallel. The ions that have passed along the first parallel region are then subjected to a second condition at the tip of the domed electrode in which the width of the spacing between the inner and outer electrodes (LTD) was variable as indicated in Figure 1. The LTD was varied from about 0.15 to 0.45 $\mathrm{cm}$. The total ion pathway length from the ion inlet orifice to the ion outlet was about $3.5 \mathrm{~cm}$. A gas flow of about $1 \mathrm{~L} / \mathrm{min}$ carried the ions along the analyzer region, and transported the ions into the vacuum system of an ion trap mass spectrometer (Thermo Electron LCQ Deca XP Max, San Jose, CA).

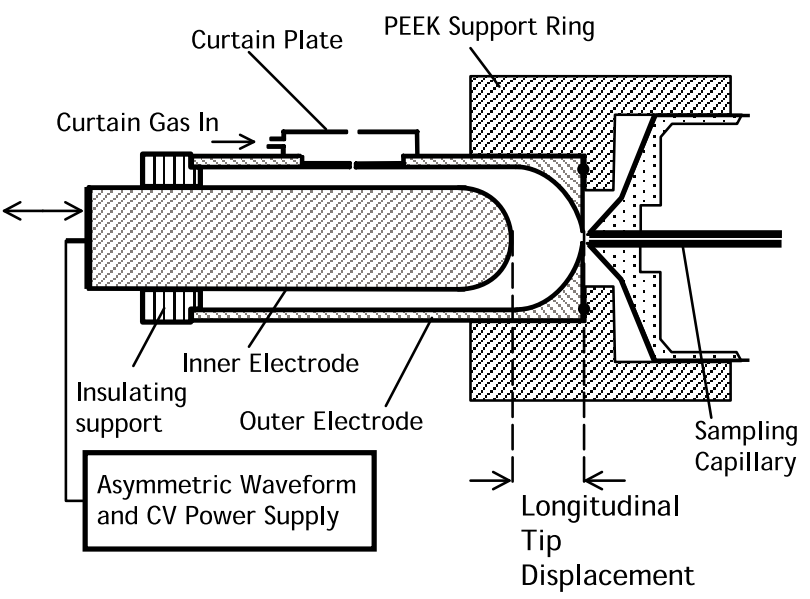

Figure 1. Cylindrical FAIMS with a dome tipped inner electrode supported by a PEEK mounting ring with gas-tight connection to the ion inlet of a Thermo Electron LCQ Deca XP Max ion trap mass spectrometer. The position of the hemispherical tip of the inner electrode was adjustable relative to the outer electrode.

As shown in Figure 1, the inner cylinder of FAIMS is terminated as a hemisphere with the same radius as the cylindrical portion of the electrode. The end of the outer cylinder is also hemispherical in shape and has an ion outlet opening of about $1 \mathrm{~mm}$ at its center, with razor sharp edges. In the FAIMS device shown in Figure 1 a longitudinal tip displacement within the range of 0.17 to $0.25 \mathrm{~cm}$ was typically required for optimum operation of FAIMS. Since the surfaces at the end of the electrodes are curved to form a spherical geometry, the electric fields in the radial direction (relative to the center of the hemisphere at the tip of the inner electrode) are not identical to those along the annular, cylindrical geometry, analyzer region. For this reason, the longitudinal location of the inner electrode was adjustable and the optimum location was established empirically.

The radial variation in electric field strength in the region of spherical geometry differs from that in the cylindrical geometry region of FAIMS. The behavior of the ions in both FAIMS analyzer regions was modeled with numerical processing techniques described previously [27-30]. This technique was used to calculate the distribution of the ions between the electrode surfaces and to calculate the time-dependent changes in ion density following ion introduction due to the loss of ions that occurs when a portion of the ion distribution is in contact with an electrode surface.

For this study, the ions were generated by electrospray ionization using the ESI source provided on the Thermo Electron LCQ Deca XP Max mass spectrometer. A fine tipped capillary with a concentric flow of nebulizer gas (nitrogen at $300 \mathrm{~mL} / \mathrm{min}$ ) was held at $3800 \mathrm{~V}$ relative to the FAIMS curtain plate. A solution of $2 \mathrm{mM}$ cesium iodide in $\mathrm{MeOH}$ was delivered at a flow rate of $1 \mu \mathrm{L} / \mathrm{min}$ to the electrospray capillary by a syringe pump. The FAIMS curtain plate was held at $1000 \mathrm{~V}$ more positive than the outer FAIMS electrode to assist 
in ion transmission across the desolvation region of FAIMS. A flow of purified dry nitrogen curtain gas (3.5 $\mathrm{L} / \mathrm{min}$ ) was provided between the FAIMS curtain plate and the FAIMS outer electrode to assist in desolvation of the ions produced by electrospray, and to minimize the entrance of droplets and neutral solvent molecules into the FAIMS analyzer. Ion separation and transmission through FAIMS is adversely affected by trace contaminants in the carrier gas stream [4].

The window of $\mathrm{CV}$ for transmission of $\mathrm{Cs}^{+}$through each FAIMS prototype was measured at different asymmetric waveform dispersion voltages (DV) from 2500 to $5000 \mathrm{~V}$ by scanning $\mathrm{CV}$ from 0 to $-60 \mathrm{~V}$ while monitoring $\mathrm{m} / \mathrm{z} 133$ at a given DV. The FAIMS prototypes were constructed with a PEEK support ring designed to be mounted in gas-tight connection onto the ion inlet capillary of the MS instrument. The outer cylinders of the FAIMS prototypes were inserted into the PEEK support ring until the outer electrode was close to the tip of the capillary. The ion source housing provided with the Thermo Electron LCQ Deca XP Max was offset from its original location by extending the support pins, and gas and electrical connections by about $2.8 \mathrm{~cm}$.

The LTD was established by measuring the length of the electrode that protruded out of the back of the electrode support, relative to a position in which the inner electrode was in contact with the outer electrode (LTD of zero length).

\section{Results and Discussion}

Figure 2 illustrates a series of compensation voltage (CV) scans for $\mathrm{Cs}^{+}$ion $\mathrm{m} / \mathrm{z} 133$ collected at various peak voltages of the asymmetric waveform (dispersion voltage, DV). From this data (296 K, 770 Torr) the field dependence of the mobility of the $\mathrm{Cs}^{+}$ion can be determined [21, 31-33]. In order to use the calculation procedures described previously, the LTD of the prototypes used in this work was set at values determined to have negligible impact on the CV peak shape. The $\alpha$ parameter was estimated as $8 \times 10^{-6} \mathrm{Td}^{-2}$ and the $\beta$ parameter to be zero. Although the numerical values of $\alpha$ and $\beta$ appear to differ between publications, these values are used to calculate the mobility at high $\mathrm{E} / \mathrm{N}$, and the various pairs of alpha, beta yield $\mathrm{K}(\mathrm{E})$ values that are comparable. Also, the parameters $\alpha$ and $\beta$ are applicable up to the limit of field strength within the data set used for their determination, and must not be used in an extrapolation to field strengths beyond that of the original data set.

The distribution of the cloud of ions between the surfaces of the inner and outer electrodes of FAIMS can be estimated based on the counter-balance of two opposing effects: a first virtual potential well that tends to confine the ions (focusing effect [11]) and a second series of effects that tend to disperse the ions (diffusion, electrostatic repulsion, gas turbulence etc.). The focusing effect is readily determined using a numerical simulation of the ion motion during one

\section{$0.4 / 0.6 \mathrm{~cm}$}

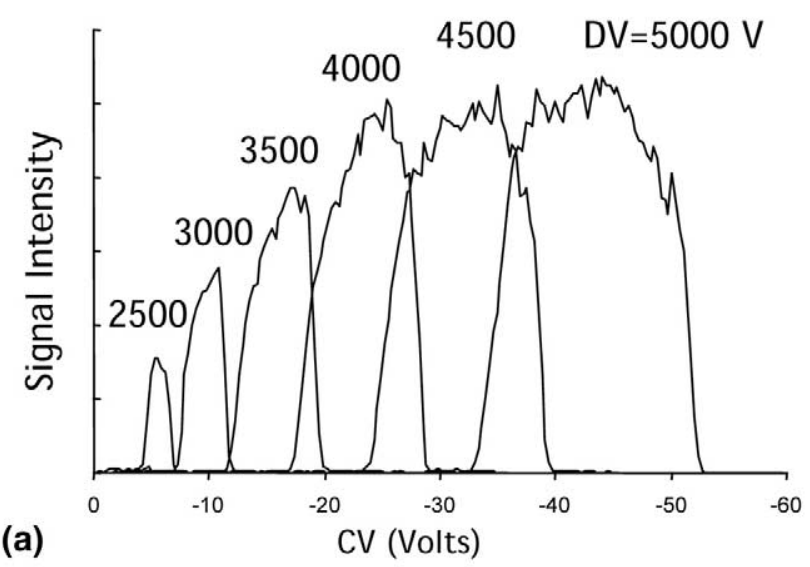

$0.2 / 0.4 \mathrm{~cm}$

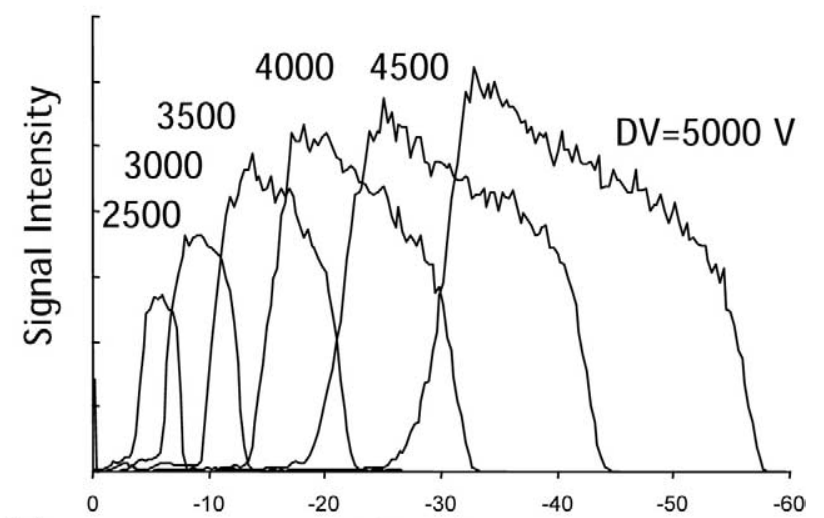

(b)

CV (Volts)

Figure 2. $\mathrm{CV}$ scans of $\mathrm{Cs}^{+}$ion at various asymmetric waveform voltages taken using domed FAIMS with inner/outer electrode radii of (a) $0.4 / 0.6 \mathrm{~cm}$ and (b) $0.2 / 0.4 \mathrm{~cm}$.

cycle of the asymmetric waveform at each radial distance within the annular space between concentric cylinders. The net displacement of the ion during one cycle of the waveform at each radial distance is translated into a virtual electric field, which is in turn is converted by integration into a virtual potential well $[27,28]$. The behavior of a cloud of ions in the time-independent virtual potential well is determined using a numerical simulation that treats the cloud as a compressible fluid [27].

Figure 3 illustrates examples of the distribution of ions calculated at two locations shown as " $\mathrm{A}$ " and " $\mathrm{B}$ " in the domed version FAIMS with inner/outer electrode radii of $0.4 / 0.6 \mathrm{~cm}$ illustrated in Figure $3 \mathrm{a}$. At location " $\mathrm{A}$ " the electrode geometry is cylindrical. At location " $B$ ", assuming that the longitudinal tip displacement is exactly equal to the electrode spacing at " $\mathrm{A}$ ", the electrode geometry is spherical. In both cases, the ion distribution was calculated as a final equilibrium time-independent state. This steady state is reached as a result of an exact balance between the 
(a)
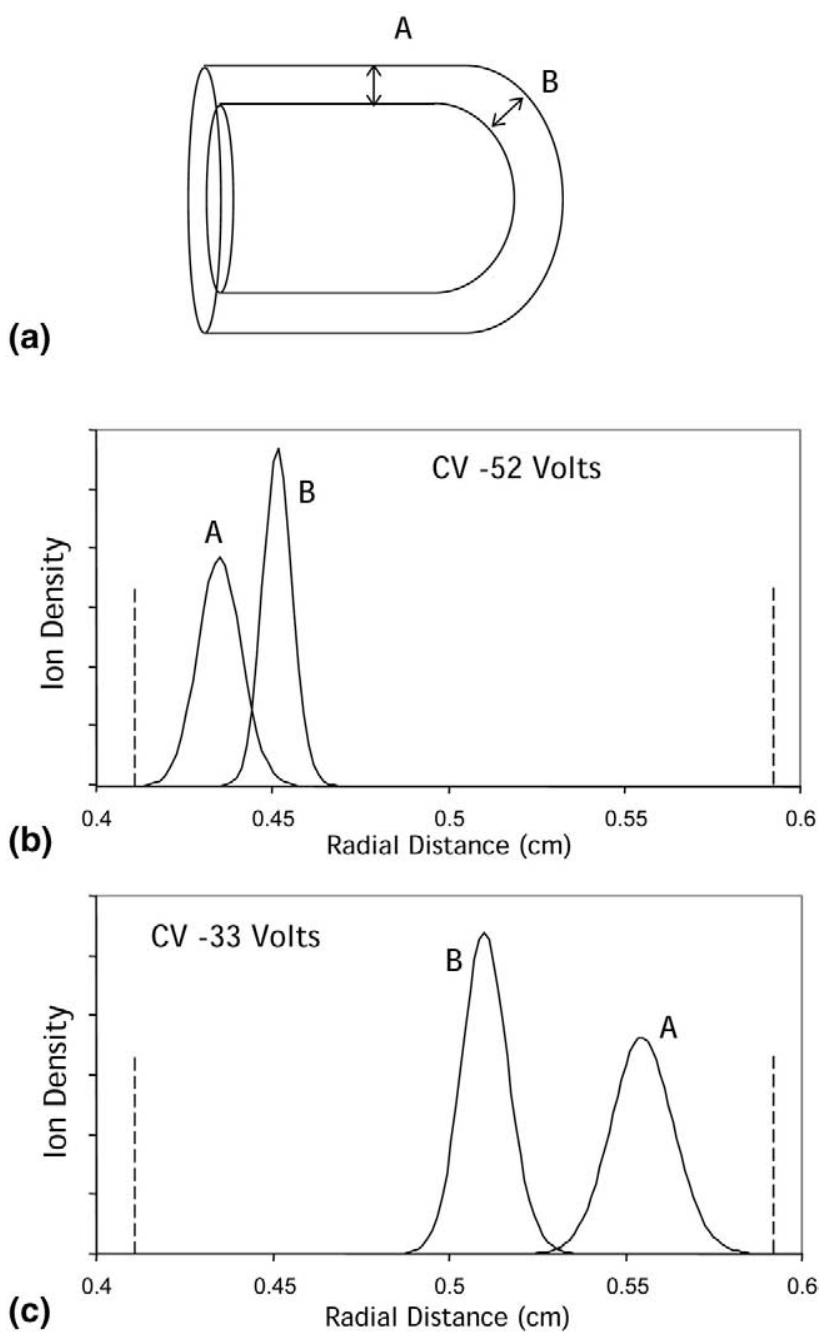

Figure 3. Calculated distribution of $\mathrm{Cs}^{+}$ion between the surfaces of a cylindrical domed FAIMS with inner/outer electrode radii of $0.4 / 0.6 \mathrm{~cm}$ in cylindrical and spherical regions shown in (a) at asymmetric waveform voltage of $5000 \mathrm{~V}$, (b) CV of $-52 \mathrm{~V}$, and (c) $\mathrm{CV}$ of $-33 \mathrm{~V}$.

confining effect of focusing and the dispersing effect of diffusion. If the ion distribution extends sufficiently widely to contact an electrode surface, ions are lost and the distribution is no longer timeindependent.

The steady state ion distribution is readily calculated once the virtual potential well has been determined using numerical techniques. For example, assume that the virtual potential well can be described by $\mathrm{V}(r)$, at some CV, DV experimental conditions for an ion of interest. If the radial ion distribution is $\mathrm{n}(r)$, the number of ions that are moving across a boundary at radial location $r$ via diffusion is computed using the coefficient of diffusion $\mathrm{D}$ and the gradient of ion density in the radial direction at location $r$. Similarly, the number of ions that cross in the opposite direction because of the focusing effect of the virtual potential well is calculated from the mobility of the ions and the strength of the virtual electric field. Setting these two quantities equal gives:

$$
\mathrm{D}\left(\frac{\mathrm{dn}(r)}{\mathrm{d} r}\right)=\mathrm{K}_{\mathrm{mob}} \mathrm{n}(r) \mathrm{E}(r)
$$

where $\mathrm{K}_{\mathrm{mob}}$ is the low field ion mobility and $\mathrm{E}(r)$ is the virtual electric field. This differential equation is solved to show that the ion distribution $\mathrm{n}(r)$ is:

$$
\mathrm{n}(r)=\mathrm{n}_{\mathrm{p}} \exp \left(\frac{-\mathrm{K}_{\mathrm{mob}} \mathrm{V}_{\mathrm{v}}(r)}{\mathrm{D}}\right)
$$

where $\mathrm{V}_{\mathrm{v}}(r)$ is the virtual potential determined from the integration of $\mathrm{E}(r)$, taking the bottom of the potential well to be at a potential equal to zero. The constant of integration is $n_{p}$, the peak ion density in the distribution $\mathrm{n}(r)$. The ratio of $\mathrm{K}_{\mathrm{mob}} / \mathrm{D}$ is equal to $\mathrm{q} / \mathrm{kT}$ at low field strength, the well-known Nernst-Townsend-Einstein relationship. In stronger fields, a generalized Einstein relation is applicable [24], which describes $K_{\mathrm{mob}} / \mathrm{D}$ in terms of coefficients of diffusion in the transverse $\left(D_{T}\right)$ and longitudinal directions $\left(D_{L}\right)$ relative to the direction of ion travel. In FAIMS the ion distribution described by eq 2 is always aligned with the longitudinal ion motion and $D_{L}$ (averaged over one cycle of the asymmetric waveform) can replace $\mathrm{D}$ in eq 2 . Since the $\mathrm{D}_{\mathrm{L}}$ in strong fields exceeds the low-field $\mathrm{D}$, the ion distribution is slightly widened. The impact of this widening of the ion distribution on the peak shapes in CV spectra is predicted to be small [22].

Figure 3 shows the calculated time-independent distribution of ions at locations $\mathrm{A}$ and $\mathrm{B}$ for an electrode set having radii $0.4 / 0.6 \mathrm{~cm}$ operated at $\mathrm{DV}=5000 \mathrm{~V}$ and at compensation voltages of -52 and $-33 \mathrm{~V}$. Figure $3 \mathrm{~b}$ shows that at $\mathrm{CV}=-52 \mathrm{~V}$ the cloud of ions is close to the inner electrode along the cylindrical portion of FAIMS, and Figure $3 \mathrm{c}$ shows that at $\mathrm{CV}=-33 \mathrm{~V}$ the cloud of ions is close to the outer electrode. In order to use eqs 1 and 2, the ion cloud must not contact either electrode. Contact with an electrode results in a timedependent exponential decay in the density of the ion cloud as the ions are neutralized by contact with the electrode. The time-constant for the exponential loss is a function of the degree that the distribution overlaps with the electrode and the derivative of this distribution near the electrode.

Figure 3 also illustrates the radial ion distributions (indicated by " $\mathrm{B}$ " in Figure $3 \mathrm{~b}$ and $3 \mathrm{c}$ ) calculated for electrodes with spherical geometry. In practice, since the ions are being transported by a flow of gas around the terminus of the inner electrode, it is possible that (1) insufficient time is available to reach time-independent equilibrium and/or (2) motion of the gas in a curved region forms eddies that distort the radial ion distribution. Nevertheless, for illustrative purposes Figure 3 shows that in both cases, CV -52 and $C V-33$, the equilibrium ion distribution would be farther from the 
electrode surfaces for the spherical geometry than for the cylindrical geometry. In other words, if the CV is more negative than $-52 \mathrm{~V}$, the ions will collide with the walls of the cylindrical portion of FAIMS, but (if they reached this far) the ions would not collide with the walls of the spherical portion. Similarly, at a CV less negative than $-33 \mathrm{~V}$, the ions collide with the outer walls along the cylindrical portion of FAIMS, but ions would not be lost if they reached the spherical portion of FAIMS. The range of $\mathrm{CV}$ for which the ion cloud remains within the electrodes is wider for the spherical geometry than for the cylindrical geometry. Similarly, Figure 3 illustrates that the focusing effect of the virtual potential well is stronger for spherical geometry than for cylindrical geometry, and the equilibrium ion distributions are narrower in the location " $\mathrm{B}$ " than " $\mathrm{A}$ " in Figure 3.

Figure 4 illustrates a comparison of an experimental measurement (Figure 4a) of the transmission of $\mathrm{Cs}^{+}$ion through FAIMS at DV $5000 \mathrm{~V}$ for electrodes with inner/outer radii of $0.4 / 0.6 \mathrm{~cm}$, and the computed CV traces for ideal cylindrical (Figure $4 \mathrm{~b}$ ) and ideal spherical geometries (Figure 4c). The computed CV traces were calculated by a method [27] that includes ion focusing, diffusion, changes in diffusion in strong fields, and a mechanical redistribution of ions that simulates gas turbulence and/or transient chemical associations of the ions.

The traces shown in Figure 4 indicate that for the specific condition in which the longitudinal tip displacement is approximately equal to the spacing in the cylindrical region of FAIMS, the width of the CV trace is controlled by the transmission of the ions as they pass along the cylindrical region of FAIMS. It is therefore expected that within a short range of LTD the peak shapes are not influenced by the longitudinal location of the inner electrode.

When the longitudinal tip displacement is modified to be significantly different from the spacing of the electrodes along the cylindrical portion of FAIMS, the $\mathrm{CV}$ traces are experimentally observed to change. Figure 5 illustrates a series of scans of $\mathrm{CV}$ taken with the inner electrode of a $0.2 / 0.4 \mathrm{~cm}$ FAIMS at various LTD positions. Similarly, Figure 6 illustrates CV scans taken at several LTD values using a $0.4 / 0.6 \mathrm{~cm}$ FAIMS. In both cases, the CV scan extends over a maximum range of voltages for transmission of $\mathrm{Cs}^{+}$when the LTD is approximately $0.2 \mathrm{~cm}$. The width of the window of CV voltages for transmission of $\mathrm{Cs}^{+}$decreases if the LTD is either increased or decreased significantly relative to 0.2 $\mathrm{cm}$.

The traces on Figures 5 and 6 reflect both the ranges of transmission of $\mathrm{Cs}^{+}$and the relative detected intensity of the ions at DV $=5000 \mathrm{~V}$ as a function of LTD. From Figure 5 the maximum $\mathrm{CV}$ window over which $\mathrm{Cs}^{+}$can be transmitted using a $0.2 / 0.4 \mathrm{~cm}$ FAIMS is from -25 to $-58 \mathrm{~V}$. The resolution $(\mathrm{CV} /$ peak width) is only about 1.2 . By decreasing the LTD to about $0.17 \mathrm{~cm}$ the resolution is increased to about 5 without a change
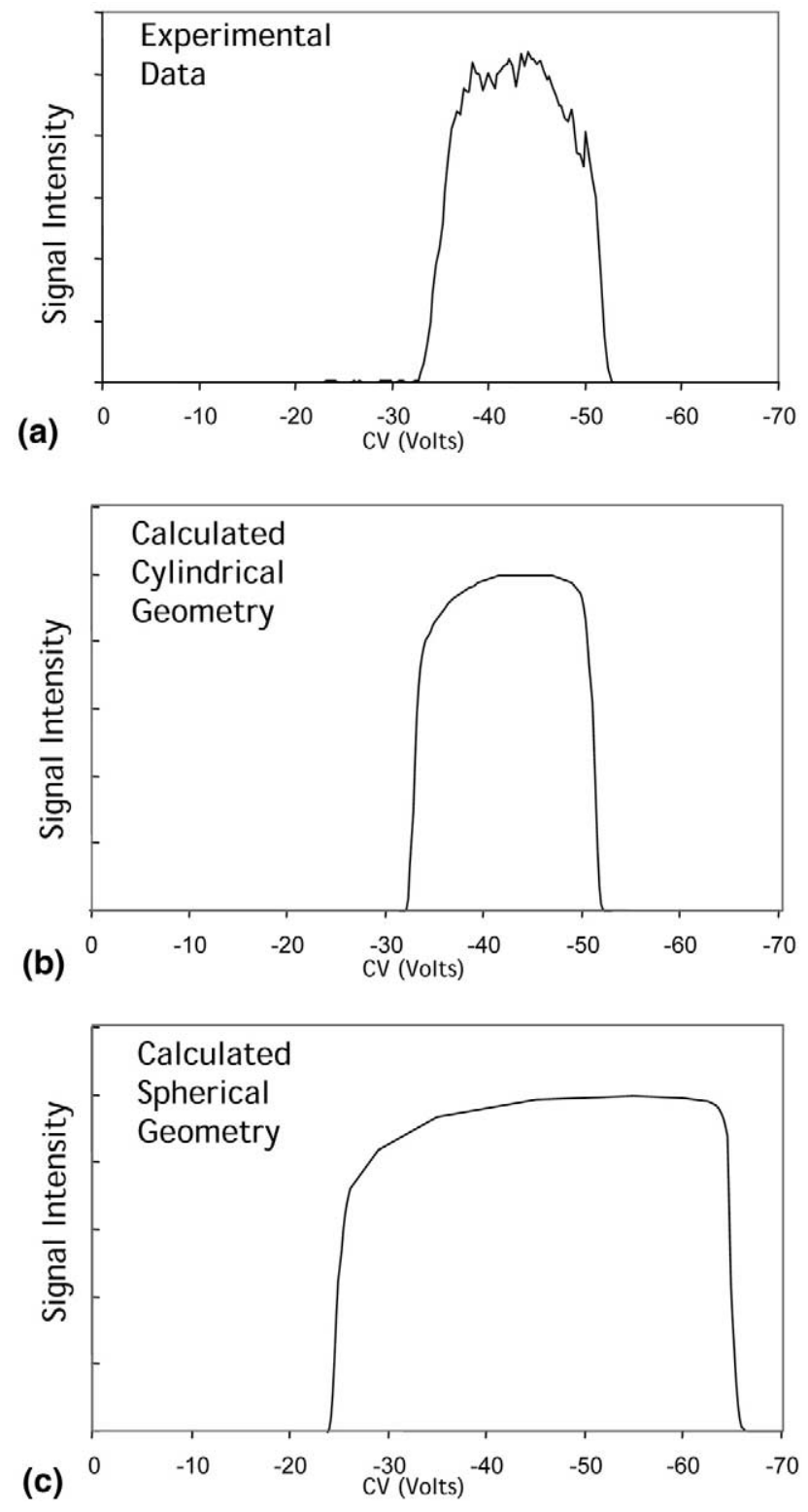

Figure 4. (a) Experimental CV scan for $\mathrm{Cs}^{+}$at DV $5000 \mathrm{~V}$ using cylindrical domed FAIMS with inner/outer electrode radii 0.4/0.6 $\mathrm{cm}$, and calculated CV scans assuming purely (b) cylindrical and (c) spherical geometry.

in sensitivity. Further decrease of LTD provides better resolution, but is accompanied by a loss of sensitivity. It appears that an increase of LTD beyond $0.3 \mathrm{~cm}$ is always accompanied by loss of sensitivity.

Figure 6 indicates that the $\mathrm{CV}$ window for $\mathrm{Cs}^{+}$ transmission using a $0.4 / 0.6 \mathrm{~cm}$ FAIMS is significantly decreased relative to the $0.2 / 0.4 \mathrm{~cm}$ FAIMS. The $\mathrm{Cs}^{+}$ ion is transmitted over a window of $\mathrm{CV}$ that ranges from -31 to $-52 \mathrm{~V}$ at a LTD of $0.2 \mathrm{~cm}$ with resolution of about 2. By decreasing LTD it is possible to increase the resolution to about 6 without sacrifice of sensitivity. In fact it is interesting to note that this decrease of LTD is somewhat accompanied by an increase of sensitivity. The CV peak shape is very sensitive to LTD when the 

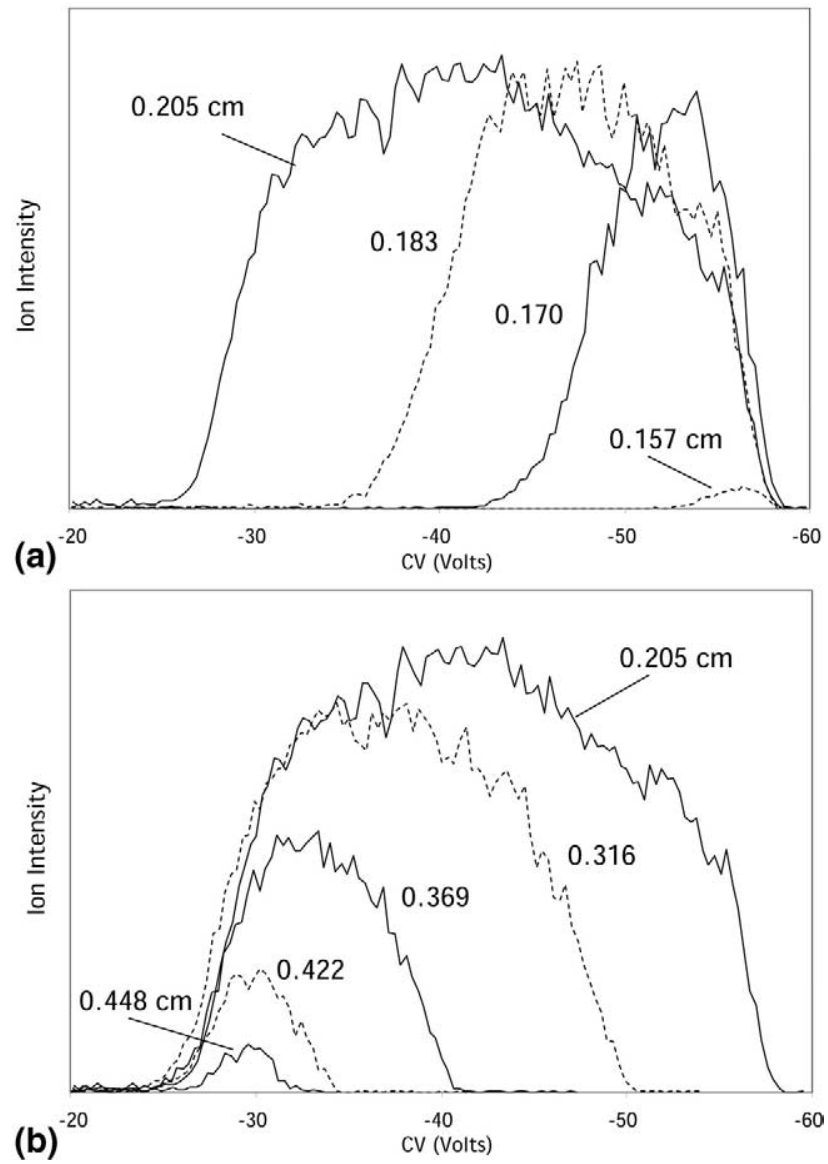

Figure 5. Experimental CV scans for $\mathrm{Cs}^{+}$at DV $5000 \mathrm{~V}$ using cylindrical domed FAIMS with inner/outer electrode radii of $0.2 / 0.4 \mathrm{~cm}$ with inner electrode longitudinally translated at (a) LTD between 0.157 and $0.205 \mathrm{~cm}$ and (b) LTD between 0.205 and $0.448 \mathrm{~cm}$.

inner electrode is translated to decrease LTD from about 0.2 to about $0.16 \mathrm{~cm}$.

\section{FAIMS Resolution Adjustment Using LTD}

Although it is beyond the scope of this report to exactly define the peak shapes for the domed FAIMS when LTD is considerably different from the analyzer width along the cylindrical portion of the electrodes, it is informative to consider the mechanism that controls the width of the peaks.

By translating the inner electrode so that LTD is wider or narrower than the normal analyzer width, the region around the tip of the domed electrode deviates from either cylindrical or spherical geometry, and the modeling of the electric fields must be done using an approach similar to that used by the ion trajectory calculation program SIMION. This calculation was described and used previously to model ion trapping in a domed FAIMS with small dimension electrodes [28]. Briefly, the fields are calculated using a numerical technique called a relaxation method which iteratively calculates the potential at a location in space by aver- aging adjacent potentials. This is repeated until the values change less than some preselected threshold. Having calculated the electric fields in the vicinity of the hemispherical dome of the inner electrode, the trajectory of an ion is calculated for one cycle of the waveform at each location. The net motion of the ion at each location is used to calculate the virtual field strength that the ion is experiencing at every such location. From this resulting virtual field, the behavior of one ion or of the entire cloud of ions can be calculated without further need for the time-dependent fields of the asymmetric waveform. The overall behavior of the ion in the time-dependent fields has been replaced by an average behavior wherein the virtual potential well is timeindependent, to provide significant simplification (and good accuracy) of the modeling of the cloud of ions.

If the LTD is exactly equal to the spacing along the cylindrical parts of the electrodes, the fields are exactly spherical and a numerical approximation of the electric fields is not necessary. At any other LTD the fields are computed with numerical methods. Figure 7a illustrates the virtual potential between the tip of the domed inner electrode (located at the right side of the figure)
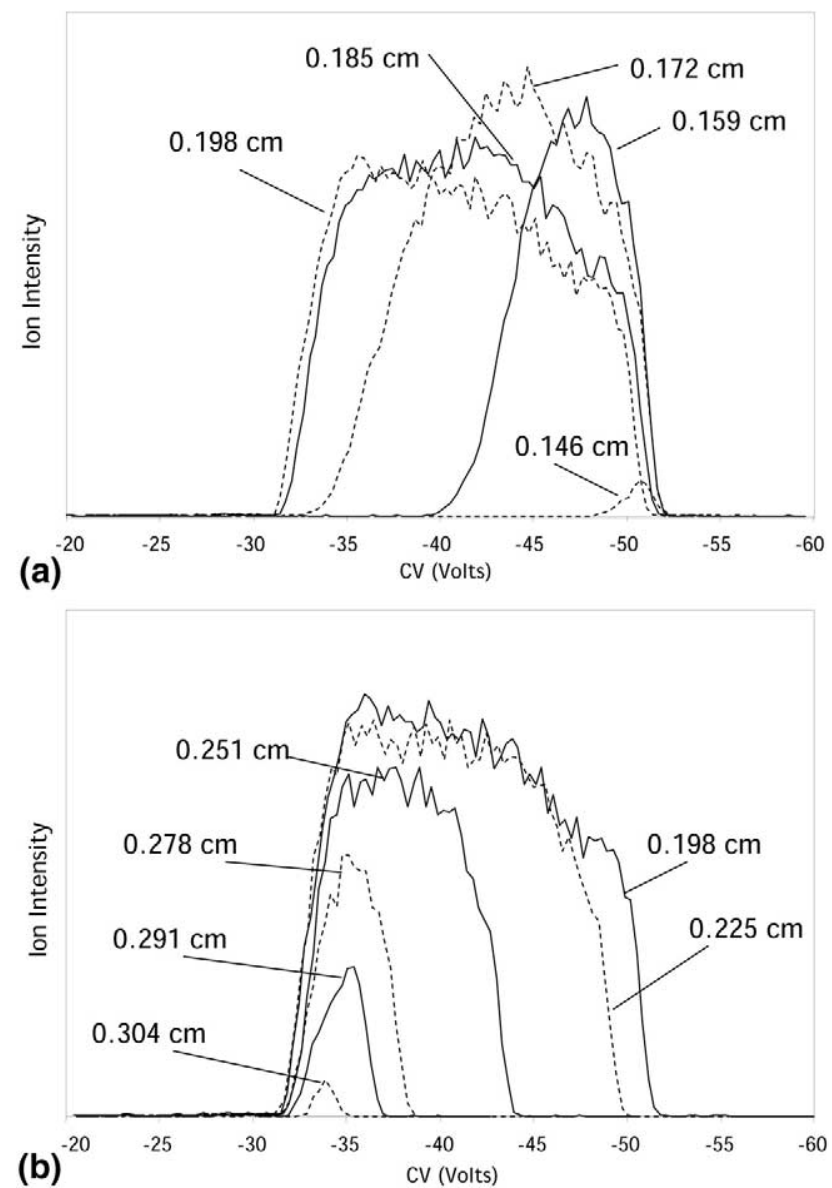

Figure 6. Experimental CV scans for $\mathrm{Cs}^{+}$at DV $5000 \mathrm{~V}$ using cylindrical FAIMS with inner/outer electrode radii of $0.4 / 0.6 \mathrm{~cm}$ with inner electrode longitudinally translated at (a) LTD between 0.146 and $0.198 \mathrm{~cm}$ and (b) LTD between 0.198 and $0.304 \mathrm{~cm}$. 


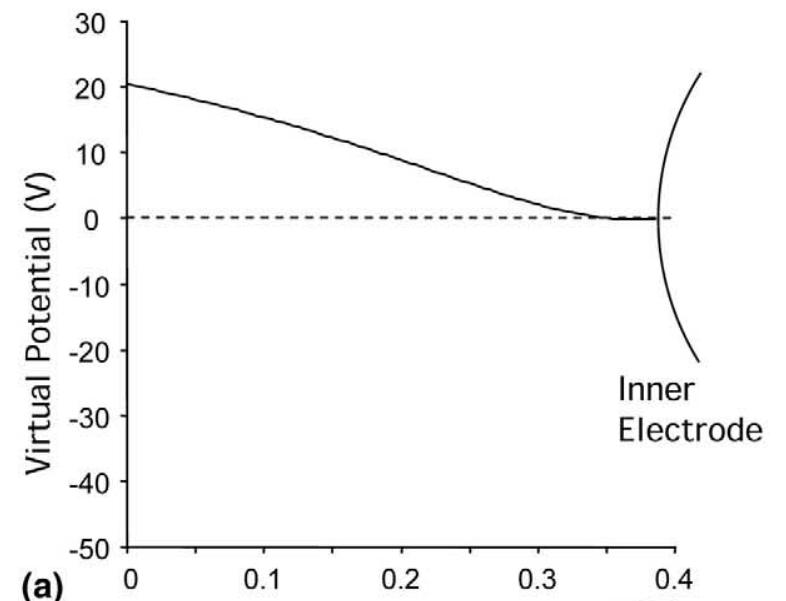

(a)
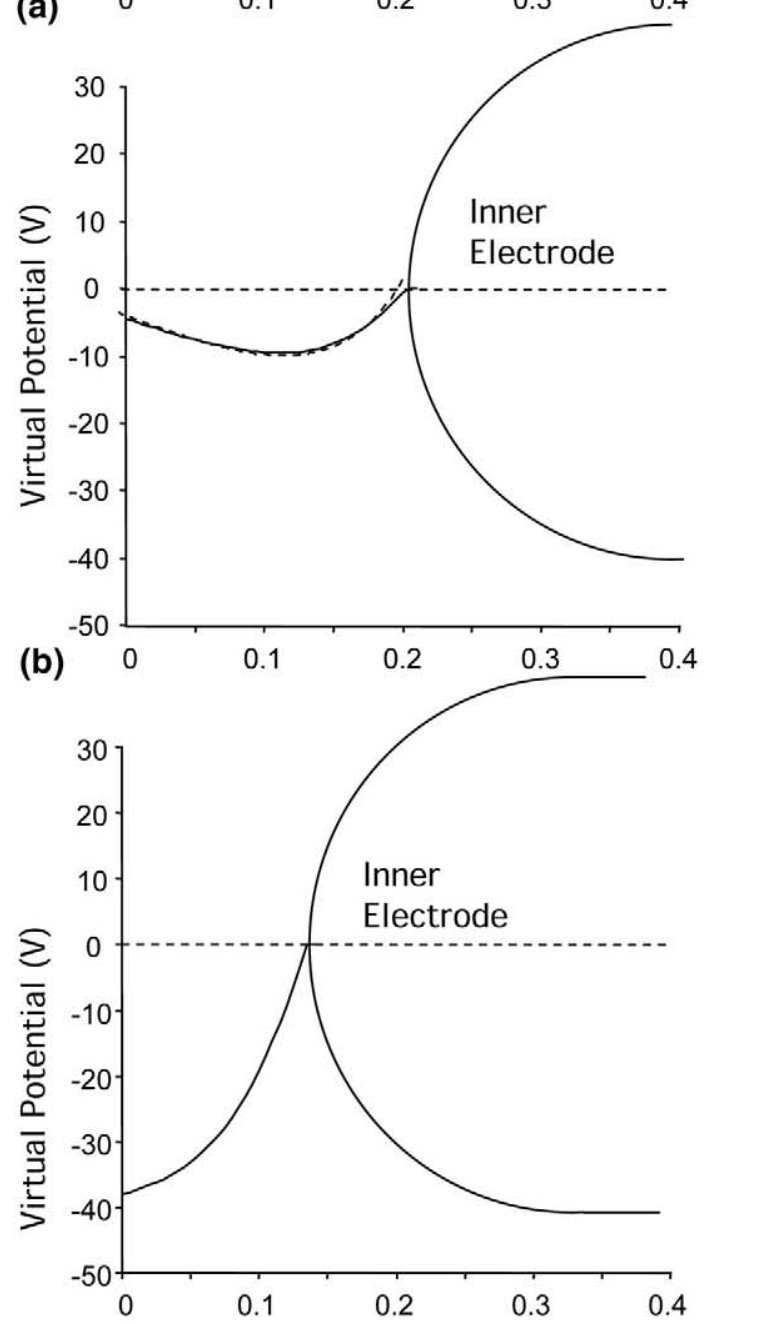

(c) Longitudinal Tip Displacement (cm)

Figure 7. Calculated virtual potential well for $\mathrm{Cs}^{+}$at DV $5000 \mathrm{~V}$ and $\mathrm{CV}-41 \mathrm{~V}$ for FAIMS with inner/outer electrode radii of $0.2 / 0.4 \mathrm{~cm}$ taken along the axis of rotation between the tip of the inner electrode and the ion exit in the outer electrode at LTD of (a) $0.4 \mathrm{~cm},(\mathbf{b}) 0.2 \mathrm{~cm}$, and (c) $0.14 \mathrm{~cm}$. Dashed line in (b) is the calculated virtual potential well for pure spherical geometry having inner/outer electrode radii of $0.2 / 0.4 \mathrm{~cm}$. and the edge of the outer electrode (left edge, at LTD = 0 ) taken along the axis of rotation of the inner electrode, for the case wherein the LTD is about $0.4 \mathrm{~cm}$ for the domed FAIMS with inner/outer electrode radii of 0.2 / $0.4 \mathrm{~cm}$. The DV of the asymmetric waveform is $5000 \mathrm{~V}$ and the $\mathrm{CV}$ is $-41 \mathrm{~V}$, corresponding to approximately mid-width of the peaks in the CV traces shown in Figure 6 for an LTD of about $0.2 \mathrm{~cm}$. In diagram Figure $7 \mathrm{a}$ the distance between the tip of the domed electrode and the outer electrode is about twice the gap of the cylindrical portion of the analyzer region, and the strength of the electric field originating from the $5000 \mathrm{~V}$ asymmetric waveform has decreased considerably relative to the cylindrical portion of the analyzer. Under these lower field conditions the expected CV for the ion to be in a "balanced" condition is very low. However, with CV of $-41 \mathrm{~V}$ applied to the inner electrode the ion is attracted to the inner electrode from every location along the distance between the inner electrode and the outer electrode along the axis of rotation. In other words, the ions are driven toward the inner electrode by a sloped "virtual potential surface" that lacks a minimum at any location between the inner and outer electrodes.

Figure $7 \mathrm{~b}$ indicates the virtual potentials along the same line as shown in Figure 7a, except with LTD of approximately $0.2 \mathrm{~cm}$, the only location where the fields are exactly spherical. Unlike the virtual potential shown in Figure 7a, a well has formed approximately mid-way between the tip of the hemispherical inner electrode and the outer electrode. Although the well is not very deep, approximately $-9.5 \mathrm{~V}$, this will focus the ions and help to prevent loss of the ions through collision with the electrode surfaces. For comparison, the dashed curve is the potential well for a spherical geometry calculated using the exact formula for the electric fields produced by the asymmetric waveform. In both cases the motion of the ion was calculated using numerical methods.

At an LTD of approximately $0.14 \mathrm{~cm}$ shown in Figure $7 \mathrm{c}$, the electric fields are considerably stronger than at the values of LTD shown in Figure 7a and $b$. The ions are forced to collide with the outer electrode by a steeply sloped virtual potential surface that lacks a minimum between the inner and outer electrodes. Figure 8 shows the virtual potentials between the inner and outer electrodes of the $0.2 / 0.4 \mathrm{~cm}$ FAIMS for a series of LTD values falling between 0.4 and $0.14 \mathrm{~cm}$. The virtual potential surfaces have been offset from each other for clarity in this figure, but all were calculated taking the tip of the inner electrode to be at a potential zero as a reference point. The deepest well in the potential surface appears at an LTD of about $0.2 \mathrm{~cm}$.

The virtual potential curves shown in Figure 8 were all calculated at a DV $=5000 \mathrm{~V}$ and $\mathrm{CV}=-41 \mathrm{~V}$, and predict the range of LTD over which ions may successfully traverse the region near the tip of the inner FAIMS electrode at this specific condition of applied voltages. This should assist in understanding of the curves 


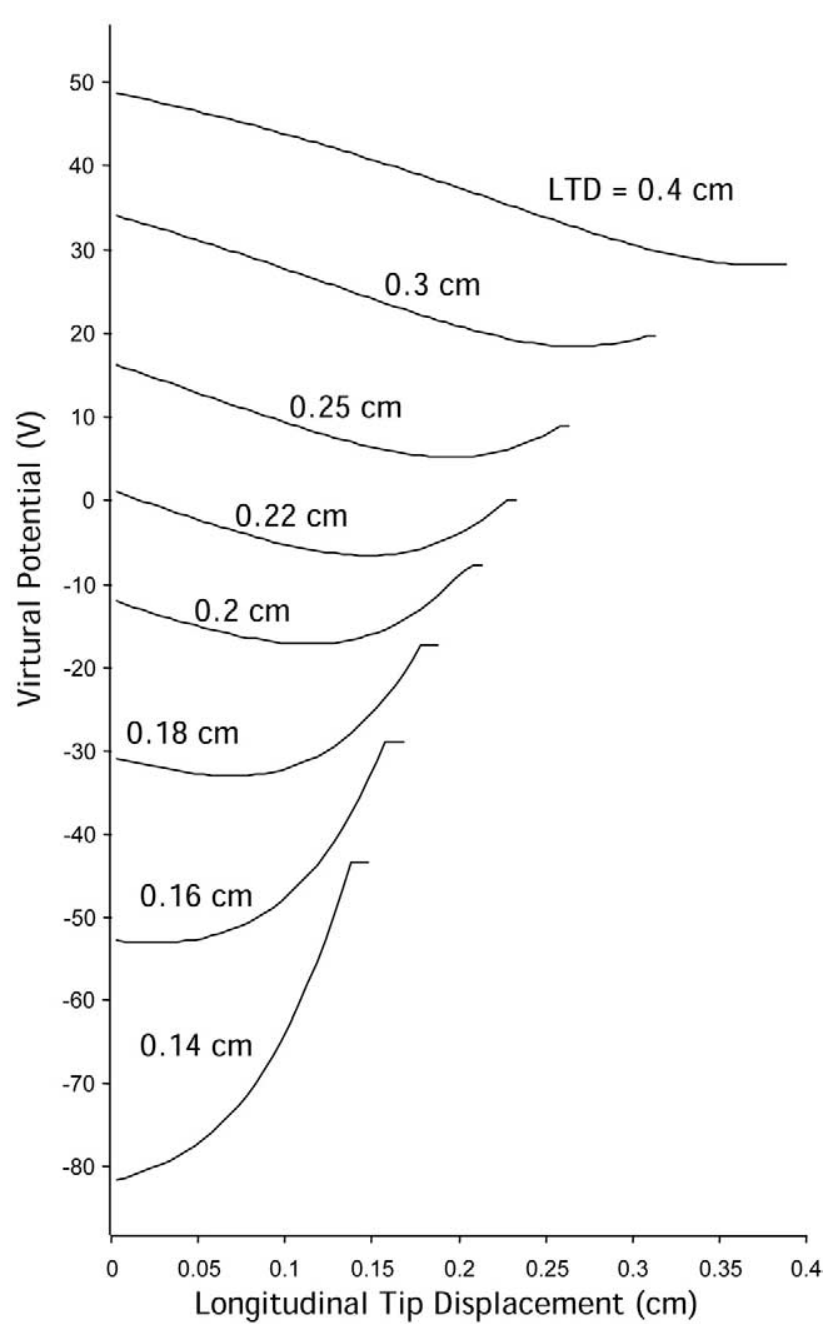

Figure 8. Calculated virtual potential well for $\mathrm{Cs}^{+}$at DV $5000 \mathrm{~V}$ and $\mathrm{CV}-41 \mathrm{~V}$ for FAIMS with inner/outer electrode radii of $0.2 / 0.4 \mathrm{~cm}$ taken along the axis of rotation between the tip of the inner electrode and the ion exit in the outer electrode at various LTD between 0.14 and $0.4 \mathrm{~cm}$. Curves have been shifted vertically for clarity. The right-hand end of each curve is adjacent to the tip of the inner electrode as shown in Figure 7, and taken to be at zero potential.

shown in Figure 6. Since Figure 8 only provides information at a single $\mathrm{CV}$ of $-41 \mathrm{~V}$, it is interesting to consider those LTD values for which some ion transmission is observed at this CV in Figure 6. For example, consider the CV peak in Figure 6 that was collected at an LTD of approximately $0.159 \mathrm{~cm}$. From the potential surface shown in Figure 8 for this LTD it might be concluded that the ions cannot pass through the region near the tip of the inner electrode of FAIMS at CV -41 $\mathrm{V}$ and LTD of $0.16 \mathrm{~cm}$ because the potential surface has no well in which to focus the ions and the transmission is expected to be low. Figure 6 confirms that this is consistent with experiment, since at an LTD of about $0.159 \mathrm{~cm}$ and $\mathrm{CV}-41 \mathrm{~V}$ the signal for $\mathrm{Cs}^{+}$is below $10 \%$ of peak intensity.

In summary, the width of the CV peaks in the experimental data in Figures 5 and 6 is a result of ion separation by the equivalent of two different FAIMS operating in tandem. The first separation occurs along the cylindrical portion of the electrodes, and a second separation occurs around the tip of the inner electrode. While the first, cylindrical portion of FAIMS cannot be altered by moving the inner electrode and the range of $\mathrm{CV}$ over which the ions are transmitted cannot be modified, the experimental window of $\mathrm{CV}$ over which ions are transmitted can be altered using the second analyzer region near the tip of the inner electrode. This second separation is experimentally controlled using LTD. The resolution of the separation of ions in FAIMS can be controlled by adjustment of the longitudinal position of the inner electrode, providing low resolution for maximization of the number of different ions that can simultaneously be observed at a single $\mathrm{CV}$, and higher resolution to improve separation of ions that would otherwise be difficult to distinguish.

\section{Conclusions}

The peak shape of the CV spectrum has been evaluated for domed type cylindrical geometry FAIMS electrodes with inner/outer electrodes of radii of $0.2 / 0.4$ and $0.4 / 0.6 \mathrm{~cm}$. By translating the inner electrode longitudinally, the shape and dimensions of the region near the tip of the inner electrode vary, and the window of CV over which ions can be transmitted also varies. The peaks in the CV spectrum become narrower as the LTD deviates from the electrode spacing along the cylindrical portion of the electrodes. The peak width of the CV spectrum is controlled by the cylindrical portion of the electrodes when LTD is approximately equal to the electrode spacing along the cylindrical portion of the electrodes. As the LTD is increased, the CV peak is truncated on the high voltage side as the stream of ions is lost by collisions with the inner electrode. When LTD is decreased, the CV peak is truncated on the low voltage side through loss by collisions with the outer electrode. The final tandem FAIMS separation is the intersection of the separation CV windows of these two differing separations, where the LTD controls the CV window of the second separation.

\section{Acknowledgments}

The authors acknowledge the contribution of Thermo Electron Corporation who provided an LCQ Deca XP Max ion trap mass spectrometer for this work. The authors thank M. Guevremont for her help during preparation of this manuscript.

\section{References}

1. Gorshkov, M. P. The Method of Impurity Analysis in Gases. USSR Inventors Certificate no. 966583; 1982.

2. Carnahan, B. L.; Tarassov, A. S. Ion Mobility Spectrometer. United States Patent 5420424; 1995.

3. Buryakov, I. A.; Krylov, E. V.; Nazarov, E. G.; Rasulev, U. K. A New Method of Separation of Multi-Atomic Ions by Mobility at Aatmospheric Pressure Using a High-Frequency Ampli- 
tude-Asymmetric Strong Electric Field. Int. J. Mass Spectrom. Ion Processes 1993, 128, 143-148.

4. Purves, R. W.; Guevremont, R.; Day, S.; Pipich, C. W.; Matyjaszczyk, M. S. Mass Spectrometric Characterization of a High-Field Asymmetric Waveform Ion Mobility Spectrometer. Rev. Sci. Instrum. 1998, 69, 4094-4105.

5. Barnett, D. A.; Ells, B.; Purves, R. W.; Guevremont, R. Separation of Leucine and Isoleucine by Electrospray IonizationHigh Field Asymmetric Waveform Ion Mobility Spectrometry-Mass Spectrometry. J. Am. Soc. Mass Spectrom. 1999, 10, 1279-1284.

6. Barnett, D. A.; Purves, R. W.; Guevremont, R. Determination of Part-per-Trillion Levels of Chlorate, Bromate, and Iodate by Eelectrospray Ionization/High-Field Asymmetric Waveform Ion Mobility Spectrometry/Mass Spectrometry. Appl. Spectrosc. 1999, 53, 1367-1374.

7. Barnett, D. A.; Purves, R. W.; Ells, B.; Guevremont, R. Separation of Ortho-, Meta-, and Para-Phthalic Acids by High-Field Asymmetric Wavefrom Ion Mobility Spectrometry Using Mixed Carrier Gases. J. Mass Spectrom. 2000, 35, 976-980.

8. Barnett, D. A.; Ells, B.; Guevremont, R.; Purves, R. W.; Viehland, L. A. Evaluation of Carrier Gases for Use in High-Field Asymmetric Waveform Ion Mobility Spectrometry (FAIMS). J. Am. Soc. Mass Spectrom. 2000, 11, 1125-1133.

9. Ells, B.; Barnett, D. A.; Purves, R. W.; Guevremont, R. Detection of Nine Chlorinated and Brominated Haloacetic Acids at Part-per-Trillion Levels Using ESI-FAIMS-MS. Anal. Chem. 2000, 72, 4555-4559.

10. Ells, B.; Barnett, D. A.; Purves, R. W.; Guevremont, R. Trace Level Determination of Perchlorate in Water Matrices and Human Urine Using ESI-FAIMS-MS. J. Environ. Monitor 2000, 2, 393-397.

11. Guevremont, R.; Purves, R. W. Atmospheric Pressure Ion Focusing in a High-Field Asymmetric Waveform Ion Mobility Spectrometer. Rev. Sci. Instrum. 1999, 70, 1370-1383.

12. Guevremont, R.; Purves, R. W.; Barnett, D. A.; Ding, L. Ion-Trapping at Atmospheric Pressure (760 torr) and Room Temperature with a High-Field Asymmetric Waveform Ion Mobility Spectrometer (FAIMS). Int. J. Mass Spectrom. 1999, 193, 45-56.

13. Guevremont, R.; Barnett, D. A.; Purves, R. W.; Vandermey, J. Analysis of a Tryptic Digest of Pig Hemoglobin Using ESIFAIMS-MS. Anal. Chem. 2000, 72, 4577-4584.

14. Guevremont, R. High-Field Asymmetric Waveform Ion Mobility Spectrometry (FAIMS). Can. J. Anal. Sci. Spectrosc. 2004, $49,105-113$

15. Venne, K.; Bonneil, E.; Eng, K.; Thibault, P. Enhanced Sensitivity in Pproteomics Analyses Using NanoLC-MS and FAIMS. PharmaGenomics 2004, 4.

16. McCooeye, M. A.; Ells, B.; Barnett, D. A.; Purves, R. W.; Guevremont, R. Quantitation of Morphine and Codeine in Human Urine Using High-Field Asymmetric Waveform Ion Mobility Spectrometry (FAIMS) with Mass Spectrometric Detection. J. Anal. Toxicol. 2001, 25, 81-87.

17. McCooeye, M. A.; Mester, Z.; Ells, B.; Barnett, D. A.; Purves, R. W.; Guevremont, R. Quantitation of Amphetamine, Methamphetamine, and their Methylenedioxy Derivatives in Urine by Solid-Phase Microextraction Coupled with Electrospray Ionization-High-Field Asymmetric Waveform Ion Mobility Spectrometry-Mass Spectrometry. Anal. Chem. 2002, 74, 30713075.

18. Purves, R. W.; Barnett, D. A.; Ells, B.; Guevremont, R. GasPhase Conformers of the $[\mathrm{M}+2 \mathrm{H}]^{2+}$ Ion of Bradykinin
Investigated by Combining High-Field Asymmetric Waveform Ion Mobility Spectrometry, Hydrogen/Deuterium Exchange, and Energy-Loss Measurements. Rapid Commun. Mass Spectrom. 2001, 15, 1453-1456.

19. Purves, R. W.; Ding, L.; Ells, B.; Guevremont, R.; Barnett, D. A. Detection of Protein Variants Using ESI-FAIMS-MS. Proceedings of the 50th ASMS Conference on Mass Spectrometry and Allied Topics; Orlando, FL, June, 2002.

20. Borysik, A. J. H.; Read, P.; Little, D. R.; Bateman, R. H.; Radford, S. E.; Ashcroft, A. E. Separation of $\beta 2$-Microglobulin Conformers by High-Field Asymmetric Waveform Ion Mobility Spectrometry (FAIMS) Coupled to Electrospray Ionization Mass Spectrometry. Rapid Commun. Mass Spectrom. 2004, 18, 2229-2234.

21. Guevremont, R. High-Field Asymmetric Waveform Ion Mobility Spectrometry: A New Tool for Mass Spectrometry. J. Chromatogr. A 2004, 1058, 3-19.

22. Shvartsburg, A.; Tang, K.; Smith, R. D. Modeling the Resolution and Sensitivity of FAIMS Analyses. J. Am. Soc. Mass Spectrom. 2004, 15, 1487-1498.

23. Shvartsburg, A.; Tang, K.; Smith, R. D. Understanding and Designing Field Asymmetric Waveform Ion Mobility Separations in Gas Mixtures. Anal. Chem. 2004, 76, 7366-7374.

24. Mason, E. A.; McDaniel, E. W. Transport Properties of Ions in Gases; John Wiley and Sons, Inc.: New York, 1988; pp 173-178

25. Rorrer, L. C., III; Guevremont, R.; Barnett, D. A.; Yost, R. A. The Role of Clustering on Ion Behavior in FAIMS. Proceedings of the 50th ASMS Conference on Mass Spectrometry and Allied Topics; Orlando, FL, June, 2002.

26. Eiceman, G. A.; Krylov, E.; Krylova, N.; Nazarov, E. G.; Miller, R. A. Separation of Ions from Explosives in Differential Mobility Spectrometry by Vapor-Modified Drift Gas. Anal. Chem. 2004, 76, 4937-4944.

27. Guevremont, R.; Purves, R. W. Comparison of Experimental and Calculated Peak Shapes for Three Cylindrical Geometry FAIMS Prototypes of Differing Electrode Diameters. J. Am. Soc. Mass Spectrom. 2005, 16, 349-362.

28. Guevremont, R.; Ding, L.; Ells, B.; Barnett, D. A.; Purves, R. W. Atmospheric Pressure Ion Trapping in a Tandem FAIMSFAIMS Coupled to a TOFMS: Studies with Electrospray Generated Gramicidin S Ions. J. Am. Soc. Mass Spectrom. 2001, 12, $1320-1330$

29. Guevremont, R.; Barnett, D. A.; Ells, B.; Purves, R. W.; Thekkadath, G. U.; Viehland, L. A. Ion Focusing in FAIMS: Ion Distribution in the Annular Space Between Cylindrical Electrodes as the Basis for Calculation of Peak Shapes in a CV Spectrum. Proceedings of the 51st ASMS Conference on Mass Spectrometry and Allied Topics; Montreal, Canada, June, 2003.

30. Guevremont, R.; Ding, L.; Purves, R. W.; Barnett, D. A. Atmospheric Pressure Trapping of Amino Acids Using a FAIMS Ion Trap Coupled to a Quadrupole/Time-of-Flight Mass Spectrometer. Proceedings of the 50th ASMS Conference on Mass Spectrometry and Allied Topics; Orlando, FL, June, 2002.

31. Guevremont, R.; Barnett, D. A.; Purves, R. W.; Viehland, L. A. Calculation of Ion Mobilities from Electrospray Ionization High-Field Asymmetric Waveform Ion Mobility Mass Spectrometry Data. J. Chem. Phys. 2001, 114, 10270-10277.

32. Viehland, L. A.; Guevremont, R.; Purves, R. W.; Barnett, D. A. Comparison of High-Field Ion Mobility Obtained from Drift Tubes and a FAIMS Apparatus. Int. J. Mass Spectrom. 2000, 197, 123-130.

33. Guevremont, R. http://www.faims.com 